\title{
Transepithelial exit of leucocytes: inflicting, reflecting or resolving airway inflammation?
}

\author{
Carl Persson, ${ }^{1}$ Lena Uller ${ }^{2}$
}

${ }^{1}$ Department of Clinical Pharmacology, Lund University Hospital, Sweden ${ }^{2}$ Department of Experimental Medical Science, Lund University, Sweden

\section{Correspondence to}

Carl Persson, Department of Clinical Pharmacology, Lund University Hospital, Lund S-22185, Sweden; carl.persson@med.lu.se

Received 14 December 2009 Accepted 2 April 2010 Published Online First 4 August 2010

\begin{abstract}
The passage of infiltrated tissue granulocytes across airway epithelium into airway lumen is generally considered to be a pathogenic process in asthma and chronic obstructive pulmonary disease (COPD). An alternative hypothesis is proposed here-namely that the transepithelial egression of infiltrated leucocytes acts to rid diseased airway tissues of proinflammatory cells efficiently and non-injuriously. Several clinical observations previously discussed as 'unexpected' and 'puzzling' support this hypothesis. In acutely resolving allergen challenge-induced inflammation, in patients with mild asthma, airway wall eosinophils disappear without evidence of apoptosis but with evidence of a developing airway lumen eosinophilia. In the same postchallenge resolution phase, lymphocytes, neutrophils and mast cells exhibit peak numbers in airway lumen of individuals with asthma. In severe asthma requiring intubation, clinical improvement is similarly parallelled by a marked increase over several days in airway lumen neutrophils. Increased numbers of sputum neutrophils and lymphocytes also occur as symptoms improve in COPD over several months after smoking cessation.

Conversely, when the transepithelial exit of leucocytes has been inhibited in inflamed animal airways the inflammation in the airway wall has been much aggravated. Finally, transepithelial egression of numerous granulocytes and lymphocytes clearly can occur without any harm to airway epithelial barriers. The present hypothesis of 'resolution through egression' provides a novel interpretation of common airway lumen data, cautions against administration of agents that impede leucocyte egression in inflammatory airway diseases and infers new approaches in disease resolution research.
\end{abstract}

\section{INTRODUCTION}

Although both the airway lumen and airway wall are designated as in vivo locations they differ dramatically. Only the wall is under the dynamic influence of nerves and microcirculation. Indeed, the human airway mucosa harbours a particularly profuse network of capillaries and venules. Throughout the nasal passages and stretching all the way from the trachea to the smallest bronchioli these microvessels occupy the area just beneath the epithelial lining. When required, the subepithelial microcirculation promptly delivers plasma proteins and leucocytes locally anywhere along the tracheobronchial tree. After extravasation the cells remain in the airway wall for variable lengths of time. Here we discuss the further fates of the airway wall leucocytes especially in association with resolution of inflammatory airways diseases.
The accessible leucocytes in the airway lumen have been extensively studied in diseases such as asthma and chronic obstructive pulmonary disease (COPD). The lumen cells are generally considered to reflect the intensity of cell-mediated inflammatory processes in the diseased airway wall. However, caution is advised in interpretation of airway lumen findings since airway wall and lumen may differ as to which granulocyte, eosinophil or neutrophil, ${ }^{1}$ and which T lymphocyte, especially Tc1 or Tc $2{ }^{2}$ is predominant. A further caveat concerns the possibility that leucocytes rise and fall in an opposite direction in the airway wall compared with the airway lumen. Here we discuss data suggesting that exit of leucocytes into the airway lumen is not the pathogenic process it is assumed to be. We argue that increased lumen leucocytes simply reflect the fact that the cells have been cleared from the diseased airway wall as inflammation resolved. This idea is not novel. In ancient medicine therapeutic purging of the airways was advocated, and authors active in $\sim 1900^{3}$ saw profuse discharge of eosinophils into sputum as a sign of recovery from severe asthma episodes.

\section{CLINICAL IMPROVEMENT AND TRANSEPITHELIAL ELIMINATION OF AIRWAY WALL LEUCOCYTES}

To some extent transepithelial migration of leucocytes into the airway lumen ('luminal entry', 'transepithelial egression', 'transmigration') is a continuous process. Several types of cells thus migrate to the airway mucosal surface to carry out immune surveillance, sentinel, defence and repair duties. For this overview we were looking for observations indicating that the transepithelial migration could also act to rid diseased airway tissues of proinflammatory cells. During development of airway inflammation newly recruited cells may accumulate in the mucosal tissue rather than moving on, into the airway lumen. But what happens when inflammation resolves? Allergen challenge in allergic subjects with asthma produces bronchial inflammation and symptoms that may resolve within $24 \mathrm{~h}$ of challenge. In such allergenchallenged subjects, ${ }^{4-6}$ and in animal models, ${ }^{7} 8$ airway lumen eosinophils have increased markedly during resolution as eosinophils evidently have disappeared from the airway wall. ${ }^{4-6}$ Hence, a negative correlation between airway wall and airway lumen cells was observed. ${ }^{4}$ Similarly, other leucocytes including lymphocytes and neutrophils have peaked in the airway lumen during the resolution phase after allergen challenge in human asthma. ${ }^{9}$ Further, increased numbers of mast cells in the airway lumen correlated with the magnitude 
of the allergen challenge-induced late phase asthma reactions recorded several hours earlier. ${ }^{10}$ There are no known data demonstrating that the airway wall inflammatory cells disappeared by mechanisms other than transmigration into the airway lumen. Thus, observations in human and animal asthma in vivo models support a resolving role of transepithelial migration of inflammatory cells.

Severe asthma is characterised by airway wall neutrophilia. ${ }^{1}$ Of interest here is that in severe asthma, requiring intubation, the number of airway lumen neutrophils increased markedly over several days as the asthma improved prior to extubation. ${ }^{11}$ Further support for a role for transepithelial egression as a mode of ridding diseased bronchial tissues of inflammatory cells comes from smoking cessation studies. Thus, symptom improvement over several months following smoking cessation in COPD was associated with sputum neutrophilia ${ }^{12} 13$ and increased sputum lymphocytes. ${ }^{12}$ Also, sputum eosinophilia has been associated with improved lung function in patients with asthma treated with $\beta$-agonists. ${ }^{14}$ Increased leucocyte numbers in the airway lumen might not be such a bad sign after all.

\section{ACCELERATED TRANSEPITHELIAL MIGRATION IN CHRONIC DISEASE}

Neutrophilia in the airway lumen in stable COPD can be associated with lack of neutrophilia in the airway wall. Similarly, patients with COPD with chronic bronchitis have been characterised by low airway wall eosinophil counts but high lumen eosinophil numbers. ${ }^{15}$ Chronic conditions such as these may thus be characterised by a degree of accelerated transepithelial migration of neutrophils and eosinophils, respectively. Stopping de novo recruitment of leucocytes to the airway wall may be an effective therapeutic intervention in these patients. In severe as compared with mild COPD, airway wall mast cells were reduced. ${ }^{16}$ This occurred without increased apoptosis of these cells but with increased numbers of mast cells in the airway lumen. ${ }^{16}$ These data, together with findings in asthma, ${ }^{10}{ }^{16}$ suggest that airway wall mast cells are eliminated through transepithelial migration.

\section{DEATH OF EOSINOPHILS AND LYMPHOCYTES IN THE AIRWAY WALL, AND STEROID TREATMENT}

Indirect support for the role of egression comes from the lack of any compelling evidence to show that airway wall leucocytes are eliminated through other mechanisms. We and others have thus failed to demonstrate a role for apoptosis, the so-called 'silent death', in resolution of airway wall eosinophilic inflammation. Airway wall eosinophils have increased and declined without occurrence of detectible apoptotic eosinophils, whether phagocytosed or not. ${ }^{717}$ Instead, we observed that primary lysis, without prior apoptosis, is a major cause of eosinophil death in vivo. ${ }^{17} 18$ This fate led to clusters of free eosinophil granules being scattered in human diseased airway mucosa. Hence, eosinophil death in vivo, through cytolysis, causes rather than resolves airway inflammation. In agreement with this, its occurrence is reduced by steroid treatment. In vitro, corticosteroids can increase apoptosis of eosinophils and lymphocytes and they may stimulate phagocytic elimination of apoptotic leucocytes. However, such acknowledged 'proresolution' effects remain to be demonstrated in the diseased airway wall. The reputed eosinophil apoptosis-inducing effect of glucocorticoids has so far not been borne out in in vivo studies on airway tissues, 781920 nor could steroid-induced T cell apoptosis be consistently demonstrated in biopsies obtained from patients with asthma ${ }^{20} 21$ and COPD. ${ }^{22}$ Apoptosis of leucocytes in the airway lumen is a different matter. However, as with the findings in the airway wall, airway lumen data may not establish the resolving role of apoptosis that we and others have been seeking (see above).

\section{IMPEDING TRANSEPITHELIAL EXIT OF LEUCOCYTES AGGRAVATES AIRWAY WALL INFLAMMATION}

It has been suggested that the induction of eosinophil apoptosis may be of benefit in allergic airway diseases. A seminal study in this field examined Fas-induced eosinophil apoptosis in the airway lumen in allergic mice. ${ }^{23}$ Later studies demonstrated that impeded transepithelial egression of granulocytes could explain the 'promising' reduction in airway lumen eosinophilia found in these Fas-treated animals. ${ }^{24}$ Many apoptotic inflammatory cells that accumulated in the airway wall were not engulfed and underwent necrosis. Indeed, airway wall inflammation was not improved by the induced apoptosis but was much aggravated. ${ }^{24}$ Inhibitors of intercellular adhesion molecule-2 (ICAM-2) ${ }^{25}$ or matrix metalloproteinase-2 (MMP-2) and MMP-926 have also reduced transepithelial migration of leucocytes and caused aggravated, and even lethal, pulmonary effects in allergic mice. Viral infection causes exacerbations of asthma and COPD. Hence, it is of interest that in rats with pre-existing virusinduced inflammation allergen challenges produced persistent eosinophilia in the airway wall but not in the airway lumen, and was associated with loss of lung elastic recoil. ${ }^{27}$ In contrast, allergen-exposed control rats had fewer eosinophils in the airway wall but more in the airway lumen and no loss of elastic recoil. ${ }^{27}$ Hence, viral infections may impede transepithelial egression of inflammatory cells, thus worsening the effects of allergen exposure. Virus-induced inhibition of egression, a major component of host defence, would also have promoted the virus infection.

Giving an antibody to block interleukin 5 (IL-5), a regulator of eosinophilopoiesis, eosinophil migration and eosinophil survival, eliminated both blood and airway lumen eosinophilia but had little effect on airway wall eosinophil numbers. ${ }^{28}$ Since no proapoptotic effect has been demonstrated, the persistent airway wall eosinophilia could well reflect attenuated egression. Recent attempts to produce beneficial effects in COPD and asthma by reducing the traffic of granulocytes include the use of chemokine receptor (CCR) inhibitors, but the possibility that such drugs may fail due to reduced egression of leucocytes from the diseased airway wall probably needs to be considered.

\section{MUCOSAL TISSUE CHEMOATTRACTION REDUCES EGRESSION BY RETAINING LEUCOCYTES IN THE AIRWAY WALL}

Airway wall neutrophilia occurs following steroid treatment of $\mathrm{COPD}^{29-31}$ and asthma. ${ }^{32-34}$ Based on findings in vitro in $1995,{ }^{35}$ this frequent observation has been attributed to inhibition of neutrophil apoptosis. Morphological features best define apoptosis, so it is significant that Gizycki et a ${ }^{29}$ were unable to find any effect of steroid treatment on neutrophil apoptosis compared with placebo treatment when studying the ultrastructure of neutrophils in COPD biopsy tissues. These authors concluded that the functional significance of the potential for steroids to reduce the clearance of neutrophils by their effect on apoptosis is unclear in vivo and requires further study'. Rather than modulating neutrophil apoptosis, we suggest the published data indicate that steroids reduce transepithelial egression of neutrophils (in contrast, steroid treatment permits transepithelial exit of eosinophils). ${ }^{7}$ In mild asthma, steroid 
treatment has caused upregulation of neutrophil-attracting chemokines such as IL-8 and interferon $\gamma$-inducible protein 10 in bronchial tissues. ${ }^{32}$ In severe exacerbations of asthma, CXCL5dependent mechanisms, possibly induced by steroid treatment, may also contribute to recruiting and retaining the neutrophils in the airway wall. ${ }^{34}$ Finally, in contrast to the steroid-induced airway wall neutrophilia, reduced numbers of airway lumen neutrophils have been demonstrated following steroid treatment of neutrophilic airway conditions. ${ }^{36-39}$ One study involving steroid-treated patients with COPD has actually demonstrated both reduced neutrophils in the airway lumen and increased neutrophils in the airway wall. ${ }^{31}$ Together these clinical findings suggest that steroid treatment reduces transepithelial egression of neutrophils and that this occurs through upregulation of mucosal tissue chemoattractants. Severe exacerbations of COPD exhibit up to a 100-fold upregulation of neutrophil chemoattractants in bronchial mucosal tissues. ${ }^{40}$ Neutrophil chemoattraction in the airway lumen in COPD may be low and neutrophils in severe COPD exhibit reduced chemotaxis compared with neutrophils in mild COPD. ${ }^{41}$ Thus, several factors may act in concert to modulate transepithelial egression of neutrophils in severe COPD.

Although inhaled steroids have broad anti-inflammatory effects and may in some cases worsen respiratory infections, they appear to spare several innate immune responses of airway microcirculation and epithelium. ${ }^{42} 43$ Steroids may also help maintain innate immunity by not reducing the profuse traffic of neutrophils to the airway epithelium and surface at viral infection and associated epithelial injury-repair processes. ${ }^{44}$ Nitrogen dioxide, a major air pollutant, is associated with an increased susceptibility to respiratory infection as well as with loss of airway wall neutrophils. ${ }^{45}$ The latter effect may reflect increased transepithelial egression since neutrophils in bronchial wash fluids were increased whereas neutrophils in bronchial epithelium were reduced after exposures to nitrogen dioxide. ${ }^{45}$ Hence, effects on airway neutrophils are difficult to interpret in simple terms of inflammation. Further studies are warranted to determine the significance of steroid-induced airway wall neutrophilia in asthma and COPD.

\section{EGRESSION OF LEUCOCYTES WITHOUT HARMING THE EPITHELIAL LINING}

The passage of granulocytes across the epithelium in asthma and COPD is thought to cause epithelial derangement of pathogenic importance. ${ }^{46} 47$ This view is debatable. For example, reduced bioelectrical resistance of epithelial cultures, commonly considered evidence of transmigration-induced barrier derangement, cannot be equated with physiologically important barrier functions in vivo. ${ }^{48}$ Also, epithelial transmembrane junction proteins, including occludin and E-cadherin, can be lost at transmigration of neutrophils without causing increased permeability. ${ }^{49}$ Finally, association between granulocytes and epithelial injury may not indicate cause and effect but simply reflect the potent stimuli that damaged cells provide for recruiting activated neutrophils and eosinophils to the repair site and to the airway lumen. ${ }^{44} 50$

The airway epithelium favours the passage of leucocytes in the physiological basal to apical direction ${ }^{475152}$ and there is ample evidence of non-injurious transepithelial migration of numerous leucocytes. In anaesthetised guinea-pigs, 35000 eosinophils $/ \mathrm{min} / \mathrm{cm}^{2}$ surface area thus traversed a normal pseudostratified tracheal epithelium without evidence of damage even at the ultrastructural level. ${ }^{51}$ Bronchial instillation of chemoattractants such as leukotriene B4 and bacterial lipopolysaccharide in humans and sheep was associated with marked movement of neutrophils into the airway lumen without epithelial injury. The yielding plasticity of paraepithelial junctions clearly allows entry of cells and macromolecules into the airway lumen without increasing epithelial permeability in the reverse direction. ${ }^{42}{ }^{53}$ Lymphatic transport of infiltrated leucocytes and extravasated plasma proteins occurs but is quantitatively of less importance than the egression-exudation processes. ${ }^{7}{ }^{42}$

\section{MECHANISMS INVOLVED IN TRANSEPITHELIAL EGRESSION}

Little is known about the molecular regulation of transepithelial loss of leucocytes during the resolution of airway inflammation. Extrapolating the extensive knowledge of mechanisms concerning the passage of leucocytes across the venular endothelial lining to mechanisms regulating the transepithelial egression of leucocytes is unwise since the two crossings are in opposite directions: the venular paraendothelial exit is apical to basal and the epithelial passage is basal to apical. Based on interactions between neutrophils and intestinal epithelial cells and focusing on pathobiology, Chin and Parkos ${ }^{47}$ have reviewed molecular mechanisms of epithelial transmigration of leucocytes. Many different adhesive interactions between neutrophils and epithelial basolateral receptors and intercellular junction proteins occur during transepithelial migration. However, details regarding neutrophil transmigration may not apply to other leucocytes. Also, intestinal epithelial mechanisms may not be entirely valid for airway epithelium. Transmigration of neutrophils may thus harm intestinal but not airway epithelial monolayers. ${ }^{49}$ Some general features of transepithelial migration of leucocytes are outlined, however, in figure 1.

MMPs may produce transepithelial loss of parenchymal granulocytes in mice by creating transepithelial chemokine gradients. ${ }^{26}$ Lumen levels of CCL11 may contribute to noninjurious loss of airway wall eosinophils, ${ }^{54} 55$ and tumour necrosis factor $\alpha$ (TNF $\alpha$ ) may promote transmigration of both eosinophils and neutrophils. ${ }^{55}$ It is widely assumed that the clearance of CCR7+ memory and effector lymphocytes from inflamed airway tissues involves apoptosis, thus 'ignoring a potentially very important exit pathway across the bronchial epithelial barrier' according to Porter and colleagues. ${ }^{52} 56$ They suggested that polarised localisation of CXCL10 ${ }^{57}$ and CXCL11 towards the apex of epithelial cells could be key chemokines for elimination of $\mathrm{T}$ lymphocytes through egression in COPD. ${ }^{52}$ Apical epithelial CCL5 ${ }^{58}$ and reduced tissue expression of CCL5 ${ }^{8}$ may regulate transepithelial migration of leuocytes in allergic airways. 'Chemorepellents' could also contribute to moving leucocytes away from sites of airway wall inflammation. ${ }^{59}$

\section{ELIMINATION OF LEUCOCYTES FROM THE AIRWAY LUMEN}

The potential role of egression as a means of reducing airway inflammation underscores the importance of cell clearance from the bronchial lumen. Death through apoptosis, followed by phagocytosis, is the topical mechanism of cell elimination. Apoptosis of cells in the airway lumen has been much studied during the last two decades with the assumption that observation on lumen cells would be valid for cells in the airway wall. However, apoptosis of lumen cells cannot reflect apoptosis in the airway wall ('dead cells do not walk'). In this overview we discuss cell apoptosis in the airway lumen merely to see if there are data indicating involvement of this mechanism in final elimination of the cells already eliminated from the airway wall. A widely quoted, uncontrolled study from $1996^{60}$ reported that steroid 


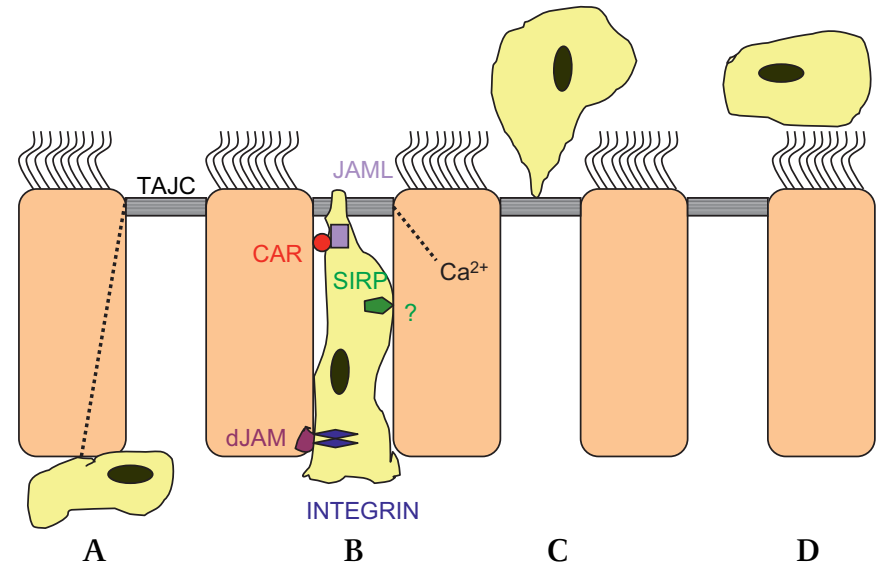

Figure 1 Sequential events in transepithelial exit of airway wall leucocytes. Modified from Chin and Parkos, ${ }^{47}$ Chun and Prince ${ }^{49}$ and Erjefält et al. ${ }^{51}$ (A) Gradients of chemoattractants across the epithelial lining would ultimately regulate the transepithelial migration of leucocytes. Chemoatttraction, perhaps involving chemoattractants released from the base of activated epithelial cells, causes migration of interstitial leucocytes towards the epithelium. The leucocytes penetrate the epithelial basement membrane probably through preformed portals (not illustrated). Different leucocyte adhesion molecules then interact with the basolateral surface of epithelial cells. This cell-cell contact evokes signalling events of potential importance for further transmigration. (B) Chemoattractant gradients across the epithelial lining may be produced by polarised epithelial apical localisation/release of chemokine ligands and involving chemokine expression mediated by cytosolic $\mathrm{Ca}^{2+}$ fluxes. The paracellular crawling of leucocytes towards the lumen may begin by leucocyte integrin binding to desmosomal junctional adhesion molecules (dJAMs). Several binding interactions and cellular signalling events (through signal-regulating proteins, SIRPs) are involved as the leucocyte crawls the relatively long distance between juxtapositioned columnar epithelial cells. Binding interactions involving JAM-like proteins (JAMLs) and specific receptors such as CAR (coxsackie and adenovirus receptor) allow the leucocyte to negotiate the tight apical junction complex (TAJC). (C) The leucocyte maintains a protein-tight seal during passage through the TAJC. Intracellular $\mathrm{Ca}^{2+}$ fluxes may cause subtle changes in the TAJC, including calpainmediated loss of occludin and E-cadherin, to facilitate leucocyte transmigration without disrupting the barrier function. At clearance of airway wall leucocytes in vivo the TAJC seal also seems perfectly intact immediately after a completed transmigration event. (D) The leucocytes leaving the airway wall mix with epithelial surface liquids. Thus, they can be readily removed by fluid flow and the mucociliary escalator. It is not known to what extent apoptosis and phagocytosis of transmigrated leucocytes contribute to final removal of lumen leucocytes. Leucocytes traversing the epithelial lining in immune surveillance, defence and epithelial repair may bind to the apical surface of epithelial cells (not illustrated) and only after some time be released into the lumen.

treatment increased the percentage of apoptotic eosinophils in the airway lumen, claiming that this action could resolve airway inflammation in asthma. A subsequent placebo-controlled trial, involving patients with asthma and administration of high dose inhaled steroid, found no increase in apoptotic eosinophils in sputum samples, however, despite a reduction in sputum eosinophils. ${ }^{61}$ Furthermore, phagocytosis was not increased; macrophages that had ingested eosinophils were rather reduced in steroid-treated individuals. ${ }^{61}$ Inconclusive clinical observations of leucocyte apoptosis in the airway lumen have also been reported in asthma, ${ }^{7} 60{ }^{6} \mathrm{COPD}^{7}{ }^{62}$ cystic fibrosis ${ }^{63} 64$ and bronchiectasis. ${ }^{65}$ Downey et al ${ }^{64}$ noted that the data showing reduced neutrophil apoptosis in resolving cystic fibrosis 'seem counter intuitive as it should be expected that neutrophil apoptosis should increase to aid resolution of infection and inflammation'. MatuteBello and Martin, ${ }^{66}$ who originally discovered an antiapoptotic action of bronchoalveolar lavage (BAL) fluid in adult respiratory distress syndrome, argued that neutrophil apoptosis may have little to do with outcome. A study testing the hypothesis that the airway lumen milieu in COPD would promote neutrophil survival failed to do so. ${ }^{62}$ In summary, the challenge of demonstrating a role for apoptosis and phagocytosis of leucocytes remains, whether in airway lumen or in the airway wall.

Leucocyte-driven airway inflammation primarily concerns the activity of leucocytes in the airway wall. Whereas clearing cells across the nasal and bronchial epithelium is likely to be beneficial for most patients, this may not be true for egression across the alveolar epithelium. However, in studies of lung inflammation in mice it appears that egression of parenchymal leucocytes into the alveolar air space was beneficial and, when transepithelial egression was prevented, severe asphyxia resulted. ${ }^{26}$ Cells can also move from the airway lumen and across the alveolar, but not bronchial, epithelium to enter the lung circulations. ${ }^{67}$ This escape route for lumen leucocytes may be important for alveolar clearance where mucociliary transport is lacking and coughing ineffective. Egression may not be desirable in diseases where mucociliary clearance is severely impaired. It is particularly important that human peripheral airways are not overwhelmed by leucocyte- and plasma protein-rich exudates that could ruin an already compromised ciliary clearance mechanism and cause small airway closure. ${ }^{42}$ Employment of non-pharmacological and pharmacological methods to improve airway clearance may be a major component of treatments aimed at resolution of airway inflammation.

\section{CONCLUSION}

We argue that the finding of eosinophils, neutrophils, lymphocytes and mast cells in the airway lumen may reflect their successful and non-injurious elimination away from cell-mediated disease areas in the airway wall. Experimental evidence and many clinical observations support the importance of transepithelial egression as a mode of ridding diseased airway tissues of inflammatory cells. This possibility needs consideration in studies of airway diseases and when assessing the effects of drug interventions where data on airway lumen leucocytes alone may lead to paradoxical conclusions. Egression-promoting drugs, combined with improved mucociliary clearance, may well have a valuable role in resolving airway inflammation in asthma and COPD.

Acknowledgements We are indebted to Anne Tattersfield and Sandra Anderson for numerous helpful suggestions on presentation of this review.

Funding Swedish Medical Research Council and Swedish Heart-Lung Foundation. Competing interests None.

Provenance and peer review Not commissioned; externally peer reviewed.

\section{REFERENCES}

1. Wenzel SE. Asthma: defining of the persistent adult phenotypes. Lancet 2006;368:804-13.

2. Barczyk A, Pierzchala W, Kon OM, et al. Cytokine production by bronchoalveolar lavage T lymphocytes in chronic obstructive pulmonary disease. J Allergy Clin Immunol 2006;117:1484-92

3. Persson CG. Centennial notions of asthma as an eosinophilic, desquamative exudative, and steroid-sensitive disease. Lancet 1997;350:1021-4.

4. Aalbers R, de Monchy JG, Kauffman HF, et al. Dynamics of eosinophil infiltration in the bronchial mucosa before and after the late asthmatic reaction. Eur Respir $J$ 1993;6:840-7.

5. Frew AJ, St-Pierre J, Teran LM, et al. Cellular and mediator responses twenty-four hours after local endobronchial allergen challenge of asthmatic airways. J Allergy Clin Immunol 1996;98:133-43 
6. Brown JR, Kleimberg J, Marini M, et al. Kinetics of eotaxin expression and its relationship to eosinophil accumulation and actvation in bronchial biopsies and bronchoalveolar lavage (BAL) of asthmatic patients after allergen inhalation. Clin Exp Immunol 1998:114:137-46.

7. Uller L, Persson C, Erjefält JS. Resolution of airway disease: removal of inflammatory cells through apoptosis, egression or both? Trends Pharmacol Sci 2006:27:461-6.

8. Uller L, Lloyd CM, Rydell-Törmänen $\mathrm{K}$, et al. Effects of steroid treatment on lung CC chemokines, apoptosis and transepithelial cell clearance during development and resolution of allergic airway inflammation. Clin Exp Allergy 2006:36:111-21.

9. Lommatzsch M, Julius $\mathbf{P}$, Kuepper $\mathbf{M}$, et al. The course of allergen-induced leukocyte infiltration in human and experimental asthma. J Allergy Clin Immunol 2006:118:91-7

10. Gauvreau GM, Lee JM, Watson RM, et al. Increased numbers of both airway basophils and mast cells in sputum after allergen inhalation challenge of atopic asthmatics. Am J Respir Crit Care Med 2000;161:1473-8.

11. Ordonez CL, Shaughnessy TE, Matthay MA, et al. Increased neutrophil numbers and IL-8 levels in airway secretions in acute severe asthma. Am J Respir Crit Care Med 2000;161:1185-90.

12. Willemse BWM, ten Hacken NHT, Rutgers B, et al. Effect of 1-year smoking cessation on airway inflammation in COPD and asymptomatic smokers. Eur Respir $\mathrm{J}$ 2005;26:835-45

13. Louhelainen $\mathbf{N}$, Rytilä $P$, Haahtela $T$, et al. Persistence of oxidant and protease burden in the airways after smoking cessation. BMC Pulmon Med 2009:9:25.

14. Green RH, Brightling CE, Wardlaw AJ, et al. Asthma exacerbations and sputum eosinophil counts. Authors' reply. Lancet 2003;361:1302-3.

15. Snoeck-Stroband JB, Lapperre TS, Gosman MME, et al. Chronic bronchitis subphenotype within COPD. Inflammation in sputum and biopsies. Eur Respir $J$ 2008;31:70-7.

16. Andersson CK, Mori M, Bjermer L, et al. Alterations in lung mast cell populations in patients with chronic obstructive pulmonary disease. Am J Respir Crit Care Med 2010:181:206-17.

17. Persson C, Erjefält J. Eosinophil lysis and free granules: an in vivo paradigm for cell activation and drug development. Trends Pharmacol Sci 1007;18:117-23.

18. Uller L, Andersson M, Greiff L, et al. Occurrence of apoptosis, secondary necrosis, and cytolysis in eosinophilic nasal polyps. Am J Respir Crit Care Med 2004:170:742-7.

19. Uller L, Persson C, Källström L, et al. Lung tissue eosinophils may be cleared through luminal entry rather than apoptosis: effects of steroid treatment. Am J Respir Crit Care Med. 2001:164:1948-56.

20. Druilhe A, Wallaert B, Tsicopoulos A, et al. Apoptosis, proliferation, and expression of $\mathrm{Bcl}-2$, Fas, and Fas ligand in bronchial biopsies from asthmatics. Am J Respir Cell Mol Biol 1998;19:747-57.

21. O'Sullivan S, Cormican L, Burke CM, et al. Fluticasone induces T cell apoptosis in the bronchial wall of mild tomoderate asthmatics. Thorax 2004;59:657-61.

22. Hodge S, Hodge G, Holmes M, et al. Increased airway epithelial and T cell apoptosis in COPD remains despite smoking cessation. Eur Respir J 2006;25:447-54.

23. Tsuyuki S, Bertrand C, Erard F, et al. Activation of the Fas receptor on lung eosinophils leads to apoptosis and resolution of eosinophilic inflammation of the airways. J Clin Invest 1995;96:2924-31.

24. Uller L, Rydell-Törmänen K, Persson C , et al. Anti-Fas mAb-induced apoptosis and cytolysis of airway tissue eosinophils aggravates rather than resolves established inflammation. Respir Res 2005;6:90.

25. Gerwin N, Gonzalo JA, Lloyd C, et al. Prolonged eosinophil accumulation in allergic lung interstitium of ICAM-2 deficient mice results in extended hyperresponsiveness. Immunity 1999;10:9-19.

26. Corry DB, Kiss A, Song LZ, et al. Overlapping and independent contributions of MMP2 and MMP9 to lung allergic inflammatory cell egression through decreased CC chemokines. FASEB J 2004;18:995-7.

27. Sorkness RL, Herricks KM, Szakaly RJ, et al. Altered allergen-induced eosinophil trafficking and physiological dysfunction in airways with preexisting virus-induced injury. Am J Physiol Lung Cell Mol Physiol 2007;292:L85-91.

28. Flood-Page $\mathbf{P}$, Menzies-Gow A, Phipps $S$, et al. Anti-IL-5 treatment reduces deposition of ECM proteins in the bronchial subepithelial basement membrane of mild atopic asthmatics. J Clin Invest 2003;112:1029-36.

29. Gizycki MJ, Hattotuwa KL, Barnes N, et al. Effects of fluticasone propionate on inflammatory cells in COPD: an ultrastructural examination of endobronchial biopsy tissue. Thorax 2002; 57:799-803.

30. Bourbeau J, Christodoulopoulos P, Maltais F, et al. Effect of salmeterol/fluticasone propionate on airway inflammation in COPD: a randomised controlled trial. Thorax 2007;62:938-43.

31. Reid DW, Wen Y, Johns DP, et al. Bronchodilator reversibility, airway eosinophilia and anti-inflammatory effects of inhaled fluticasone in COPD are not related. Respirology 2008;13:799-809

32. Fukakusa M, Bergeron C, Tulic MK, et al. Oral corticosteroids decrease eosinophil and CC chemokine expression but increase neutrophil, IL-8, and IFN-gamma-inducible protein 10 expression in asthmatic airway mucosa. J Allergy Clin Immunol 2005;115:280-6.

33. Nguyen LT, Lim S, Oates T, et al. Increase in airway neutrophils after oral but not inhaled corticosteroid therapy in mild asthma. Respir Med 2005;99:200-7.

34. Qiu Y, Zhu J, Bandi V, et al. Bronchial mucosal inflammation and upregulation of CXC chemoattractants and receptors in severe exacerbations of asthma. Thorax 2007;62:475-82.
35. Cox G. Glucocorticoid treatment inhibits apoptosis in human neutrophils. J Immunol 1995; 154:4719-25

36. Ozol D, Aysan T, Solak ZA, et al. The effect of inhaled corticosteroids on bronchoalveolar lavage cells and IL-8 levels in stable COPD patients. Respir Med 2005;99:1494-500

37. Barnes NC, Qiu YS, Pavord ID, et al. Antiinflammatory effects of salmeterol/ fluticasone propionate in chronic obstructive lung disease. Am J Respir Crit Care Med 2006;173:736-43.

38. Vagaggini B, Cianchetti S, Bartoli M, et al. Prednisone blunts airway neutrophilic inflammatory response due to ozone exposure in asthmatic subjects. Respiration 2007:74:61-8.

39. Alexis NE, Lay JC, Haczku A, et al. Fluticasone propionate protects against ozone-induced airway inflammation in modified immune cell markers in healthy volunteers. Environ Health Persp 2008;116:799-805.

40. Oiu Y, Zhu J, Bandi V, et al. Biopsy neutrophilia, neutrophil chemokine and receptor gene expression in severe exacerbations of chronic obstructive pulmonary disease. Am J Respir Crit Care Med 2003:168:968-75.

41. Yoshikawa T, Dent G, Ward J, et al. Impaired chemotaxis in chronic obstructive pulmonary disease. Am J Respir Crit Care Med 2007;175:473-9.

42. Persson C, Uller L. Role of plasma exudation in asthma and COPD. Clin Exp Allergy 2009;39:1626-9

43. Schleimer RP. Glucocorticoids suppress inflammation but spare innate immune responses in airway epithelium. Proc Am Thorac Soc 2004:1:222-30.

44. Erjefält JS, Erjefält I, Sundler F, Persson C. Effects of topical budesonide on epithelial restitution in vivo in quinea-pig trachea. Thorax 1995:50:785-92.

45. Blomberg A, Krishna MT, Helleday R, et al. Persistent airway inflammation but accommodated antioxidant and lung function responses after repeated daily exposure to nitrogen dioxide. Am J Respir Crit Care Med 1999:159:536-43.

46. Lukacs $\mathbf{N}$. Role of chemokines in the pathogenesis of asthma. Nat Rev Immunol 2001;1:108-16.

47. Chin AC, Parkos CA. Pathobiology of neutrophil transepithelial migration: implications in mediating epithelial injury. Annu Rev Pathol 2007;2:111-43

48. Moraes TJ, Rafii B, Niessen F, et al. Protease-activated receptor (Par)1 alters bioelectrical properties of distal lung epithelial without compromising barrier function. Exp Lung Res 2009;35:136-54

49. Chun J, Prince A. Ca ++ signalling in airway epithelial cells facilitates leukocyte recruitment and transepithelial migration. J Leuk Biol 2009;86:1-10.

50. Persson C, Andersson M, Uller L. Epithelial repair and function. In: Proud D, ed Pulmonary epithelium. Chichester: Wiley, 2008:75-88.

51. Erjefält JS, Uller L, Malm-Erjefält $M$, et al. Rapid and efficient clearance of airway tissue granulocytes through transepithelial migration. Thorax 2004;59:136-43

52. Porter JC, Falzon M, Hall A. Polarized localization of epithelial CXCL11 in chronic obstructive pulmonary disease and mechanisms of T cell egression. J Immunol 2008; 180:1866-77

53. Martin TR. Neutrophils and lung injury: getting it right. J Clin Invest 2002;110:1603-5.

54. Erjefält JS, Korsgren M, Malm-Erjefält M, et al. Acute allergic responses induce a prompt luminal entry of airway tissue eosinophils. Am J Respir Cell Mol Biol 2003:29:439-48

55. Kato $\mathbf{Y}$, Fujisawa $T$, Shibano $\mathrm{M}$, et al. Airway epithelial cells promote transmigration of eosinophils in a new three-dimensional chemotactic model. Clin Exp Allergy 2002;32:889-97

56. Porter JC. Epithelial Rho GTPases and the transepithelial migration of lymphocytes Methods Enzymol 2008;439:205-17.

57. Saetta M, Mariani M, Panina-Bordignon P, et al. Increased expression of the chemokine receptor CXCR3 and its ligand CXCL10 in peripheral airays of smokers with chronic obstructive pulmonary disease. Am J Respir Crit Care Med 2002;165:1404-9.

58. Taguchi M, Sampath D, Koga T, et al. Patterns for RANTES secretion and intercellular adhesion molecule 1 expression mediate transepithelial T cell traffic based on analyses in vitro and in vivo. J Exp Med 1998;187:1927-40.

59. Caulfield J, Fernandez M, Snetkov V, et al. CXCR4 expression on monocytes is upregulated by dexamethasone and is modulated by autologous CD3 $+\mathrm{T}$ cells. Immunol 2002;105:155-62.

60. Woolley KL, Gibson PG, Carty K, et al. Eosinophil apoptosis and the resolution of airway inflammation in asthma. Am J Respir Crit Care Med 1996:154:237-43.

61. Gibson PG, Saltos N, Fakes K. Acute anti-inflammatory effects of inhaled budesonide in asthma. Am J Respir Crit Care Med 2001;163:32-6.

62. Rytilä P, Plataki M, Bucchieri F, et al. Airway neutrophilia in COPD is not associated with increased neutrophil survival. Eur Respir J 2006:28:1163-9.

63. Watt AP, Courtney J, Moore J, et al. Neutrophil cell death, activation and bacteria infection in cystic fibrosis. Thorax 2005:60:659-64.

64. Downey DG, Bell SC, Elborn JS. Neutrophils in cystic fibrosis. Thorax 2009;64:81-8

65. Watt AP, Brown V, Courtney J, et al. Neutrophil apoptosis, proinflammatory mediators and cell counts in bronchiectasis. Thorax 2004;59:231-6.

66. Matute-Bello G, Martin TR. Science review: apoptosis in acute lung injury. Crit Care 2003:7:355-8.

67. Lehmann C, Wilkening A, Leiber D, et al. Lymphocytes in the bronchoalveolar space reenter the lung tissue by means of the alveolar epithelium, migrate to regional lymph nodes, and subsequently rejoin the systemic immune system. Anat Rec 2001;264:229-36. 